Reprod. Nutr. Dévelop., 1988, 28 (1), 203-204.

\title{
Estimation du débit sanguin mammaire chez la vache laitière par thermodilution. 1. Mise au point du matériel et des conditions de mesures
}

\author{
H. RULQuin, J. BERNABÉ (*), J. P. CAUDAL, Joëlle DUVÉRÉ
}

Station de Recherches sur la Vache Laitière, I.N.R.A., Saint-Gilles, 35590 L'Hermitage, France.

(*) Laboratoire de Recherches sur la Traite, I.N.R.A., 65, rue de Saint-Brieuc, 35042 Rennes Cedex, France.

Summary. A special apparatus was constructed to measure, mammary blood flow in dairy cows by the continuous thermodilution method. In vitro experiments indicated that accuracy and repeatability exceeded $2 \%$ only when injection flow (If) was adapted to the measured flow (Mf). So (If) had to be 4.5 to $6 \mathrm{ml} / \mathrm{s}$ to measure (Mf) from 4 to $6 \mathrm{l} / \mathrm{min}$ and at least $6 \mathrm{ml} / \mathrm{s}$ for (Mf) between 7 and $12.5 \mathrm{I} / \mathrm{min}$.

La thermodilution continue est une technique particulièrement bien adaptée à la mesure du débit sanguin mammaire des ruminants (Linzell, 1966). Son avantage principal sur des méthodes comme la débitmétrie électromagnétique (Peeters et al., 1979) est d'être utilisable pendant plusieurs années sur le même animal tout en présentant une précision et une rapidité satisfaisantes. Elle consiste à réaliser une injection à débit constant (Di) d'une solution plus froide (Ti) que le sang (Ts) et à enregistrer en aval du lieu d'injection la température du mélange " sang + solution " (Tm).

Le débit du sang (Ds) est donné par: $\mathrm{Ds}=\mathrm{Di}(\mathrm{Tm}-\mathrm{Ti}) /(\mathrm{Ts}-\mathrm{Tm})$. Employée principalement sur des chèvres, nous avons cherché à adapter cette technique, tout en l'automatisant, à la vache et à mesurer in vitro sa précision.

Matériel et méthodes. Les injections sont réalisées avec un pousse-seringue, spécialement conçu, qui assure des débits allant jusqu'à $9 \mathrm{ml} / \mathrm{s}$ avec une précision et une reproductibilité meilleures que $1 \%$. Il est constitué d'un moteur pas à pas entraînant par l'intermédiaire d'un moto réducteur le piston d'une seringue en Téflon de $200 \mathrm{ml}$. Celle-ci est munie d'une vanne 3 voies actionnée par un servo-moteur. L'aiguille d'injection, de type Tuohy, ou percée de 3 orifices latéraux orientés à $120^{\circ}$ (long. : $66 \mathrm{~mm} ; \emptyset_{\mathrm{i}-} \emptyset_{\mathrm{e}}: 1,2-1,4 \mathrm{~mm}$ ), est implantée à contre-courant du flux à mesurer. Les températures sont mesurées à l'aide de 2 sondes de platine ( 100 ohms à $0{ }^{\circ} \mathrm{C}, 0,03 \mathrm{~s}$ de constante de temps) connectées (par un montage 4 fils) à un milli-ohmmètre d'une centrale de mesure. La sonde (Øe $0,9 \mathrm{~mm}$; long. $15 \mathrm{~mm}$ ) mesurant la température du sang est scellée dans une aiguille de $10 \mathrm{~cm}$ de long $\left(\emptyset_{\mathrm{i}} \emptyset_{\mathrm{e}}: 1,2-1,4 \mathrm{~mm}\right)$; celle servant à la mesure de la température de l'infusion est placée dans la tête de la seringue. L'ensemble de ces appareils est relié à un micro-ordinateur qui commande la marche du pousseseringue (sens, vitesse), assure le remplissage de la seringue en fin d'injection, récupère les mesures de températures $(1 \mathrm{point} / \mathrm{s})$, calcule le débit, affiche puis archive les données. 
L'appareillage et les conditions de mesure ont été testés à l'aide d'un dispositif de simulation recréant la gamme des débits susceptibles d'être rencontrés sur des vaches produisant de 20 à $50 \mathrm{I}$ de lait/j. Celui-ci comprenait une pompe à débit réglable $(4,3$ à $12,5 \mathrm{l} / \mathrm{min})$ faisant circuler un liquide thermostaté à $37,5^{\circ} \mathrm{C}$ dans un tuyau rigide horizontal $\left(\emptyset_{i}: 20 \mathrm{~mm}\right.$ ) et le système de mesure. Le débit réel du fluide est mesuré par pesée en un temps chronométré. La précision de la technique est estimée par l'écart relatif entre le débit réel et la mesure, sa reproductibilité par le coefficient de variation de 6 à 10 répétitions par mesure.

Résultats et discussion. L'automatisation de notre technique permet une mesure toutes les 4 minutes. La nature du fluide (sang ou eau) circulant, le type d'aiguille d'injection, la température du liquide infusé (11-16 vs $\left.17-21^{\circ} \mathrm{C}\right) \mathrm{n}^{\prime}$ ont pas d'effets significatifs. La précision est fonction de l'adéquation entre le débit d'infusion et le flux à mesurer (interaction significative à $P<0,01$ ). Ainsi l'erreur est réduite en utilisant des débits d'infusion de 4,5 à $5 \mathrm{ml} / \mathrm{s}$ pour estimer des débits de 4 à $6 \mathrm{I} / \mathrm{min}$ et de $6 \mathrm{ml} / \mathrm{s}$ pour des débits de 12,5 $\mathrm{I} / \mathrm{min}$ (fig. 1). Dans ces conditions la précision et la reproductibilité sont toutes deux de $2 \%$. L'adaptation du débit d'infusion à celui du fluide à mesurer est sans doute nécessaire à la réalisation d'un mélange homogène des 2 liquides. Elle pourra être facilement réalisée pour la mesure du débit sanguin mammaire, chez la vache laitière, par l'estimation approximative de celui-ci à partir de la production de lait (Rulquin, 1981).

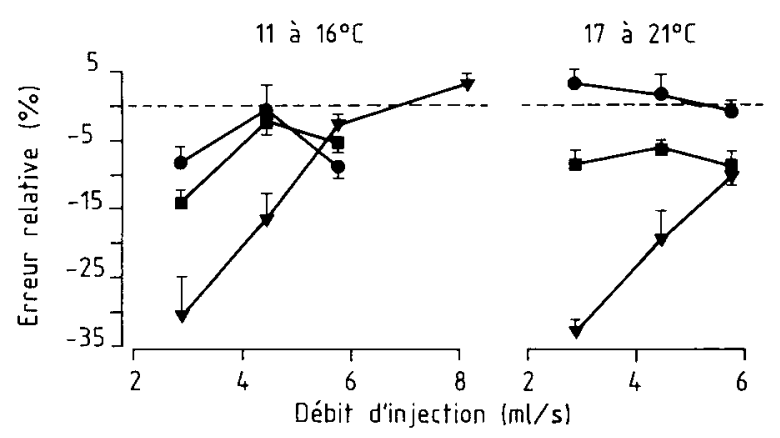

FIG. 1. - Effets de la température du liquide injecté $\left(11\right.$ à $16^{\circ} \mathrm{C}$ ou 17 à $\left.21^{\circ} \mathrm{C}\right)$ et du débit d'injection sur la précision de la mesure du débit du fluide circulant pour différentes valeurs de ce débit : 4,3 ; 6,$9 ; \nabla 12,5 \mathrm{I} / \mathrm{min}$.

Linzell J. L., 1966. Circulation Research, XVIII, 745-754.

Peeters G., Houvenaghel A., Roets E., Massart-Leen A. M., Verbeke R., Dhondt G., Vershooten F., 1979. J. anim. Sci., 48, 1143-1153.

Rulquin H., 1981. Reprod. Nutr. Dévelop., 21, 31-46. 